\title{
What Really Is Going on? Review, Critique and Extension of Situation Awareness Theory
}

\author{
Paul M. Salmon ", Neville A. Stanton, Daniel P. Jenkins, Guy H. Walker, \\ Mark S. Young, and Amardeep Aujla \\ Defence Technology Centre for Human Factors Integration (DTC-HFI) \\ Brunel University, BIT Lab, School of Engineering and Design, Uxbridge, \\ Middlesex, UK, UB8 3PH \\ paul.salmon@brunel.ac.uk
}

\begin{abstract}
Theoretically, Situation Awareness (SA) remains predominantly an individual construct. The majority of the models presented in the literature focus on SA from an individual perspective and in comparison, the concept of team SA has received less attention. SA in complex, collaborative environments thus remains a challenge for the human factors community, both in terms of the development of theoretical perspectives and of valid measures, and also in the development of guidelines for system, training and procedure design. This article presents a review and critique of what is currently known about SA and team SA, including a comparison of the most prominent individual and team models presented in the literature. In conclusion, we argue that recently proposed systems level Distributed Situation Awareness (DSA) approaches are the most suited to describing and assessing SA in real world collaborative environments.
\end{abstract}

Keywords: Situation Awareness, Teams, Collaborative Systems.

\section{Introduction}

Ostensibly, Situation Awareness (SA) refers to the level of awareness and understanding that an individual has of a situation, an operator's dynamic understanding of 'what is going on' (Endsley, 1995a). Such is its popularity, much has been written about the construct, yet there remains some disparity between the various models presented in the literature. Further, SA in complex collaborative environments requires much further investigation, despite the fact that team SA is now recognised as a critical factor in collaborative system design and assessment (Shu and Furuta, 2005). This article presents a summary of a review and critique of what is currently known on SA and team SA, following which a recently developed approach for describing SA in complex collaborative environments is presented.

\section{Situation Awareness}

First emerging as a concept of great interest in during the First World War (Press, 1986; cited in Endsley, 1995a), SA has since enjoyed great attention from the human

\footnotetext{
* Corresponding author.
} 
factors community. Following Endsley's (1995a, b) seminal work, published in a special issue of the Human Factors journal on SA in 1995, SA rapidly emerged as a prominent factor in system design, analysis and performance-based research across many complex domains. Interest in the construct continues to expand, yet it remains a contentious topic. Depending upon where you look in the literature, there are various different views on the construct and there have many attempts at defining it. The main point of contention lies in the description of SA as either the cognitive process used to develop and maintain SA, to the tangible product of SA, or to a combination of both the process used and the end product of SA. Endsley (1995a), for example, describes $\mathrm{SA}$ as a product (resulting from a process called situation assessment) comprising the following three levels:

\section{"The perception of the elements in the environment within a volume of time and space, the comprehension of their meaning, and the projection of their status in the near future" (Endsley, 1995a, p 36).}

Fracker (1991), on the other hand, describes the process of "combining of new information with existing knowledge in working memory and the development of a composite picture of the situation along with projections of future status and subsequent decisions as to appropriate courses of action to take" (Fracker, 1991). From the process-product school, Smith and Hancock (1995) describe the construct as 'externally directed consciousness' and suggest that SA is, "the invariant in the agent-environment system that generates the momentary knowledge and behaviour required to attain the goals specified by an arbiter of performance in the environment" (Smith and Hancock, 1995).

\section{Situation Awareness Theory}

Inaugural SA theories were, in the main, focused on describing the SA of individual operators (e.g. Endsley, 1995a) and the models that currently dominate the literature are individual-based theories, such as Endsley's information processing-based threelevel model (Endsley, 1995a), Smith and Hancock's perceptual cycle model (Smith and Hancock, 1995) and Bedny and Meister's (1999) theory of activity model. These three models are presented in Figure 1 and compared and contrasted in Table 1.

Endsley's model is generic and presents an intuitive description of SA, but its greatest utility lies in its simplicity and also the division of SA into three hierarchical levels, which allows the construct to be measured easily and effectively, and also supports the abstraction of SA requirements and the development of specific training strategies and design guidelines to support the acquisition of SA. Indeed none of the other models support such endeavors so effectively. The three-level model, however, is not without its flaws. For example, Endsley distinguishes between the product of SA and the processes that are used to achieve it, suggesting that the two are separate. Her definition is therefore contradictory, since it refers to the 'perception of the elements', the 'understanding of their meaning', and the 'projection of future states', all of which could be taken to be processes involved in the development of SA. Also, 


\begin{tabular}{|c|c|c|c|}
\hline 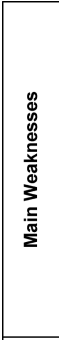 & 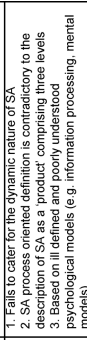 & 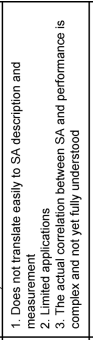 & 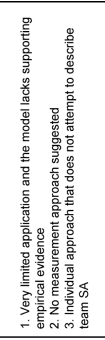 \\
\hline 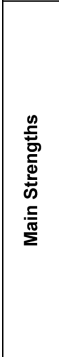 & 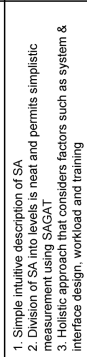 & 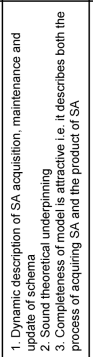 & 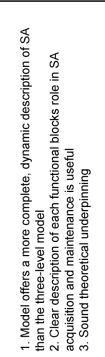 \\
\hline 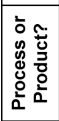 & 营 & 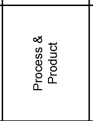 & 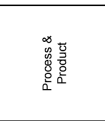 \\
\hline 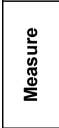 & 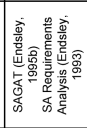 & 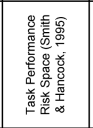 & $\frac{\underline{s}}{z}$ \\
\hline $\begin{array}{l}\text { 胥 } \\
\text { 竞 }\end{array}$ & $\frac{\mathscr{e}}{\mathrm{g}}$ & 墨 & 总 \\
\hline 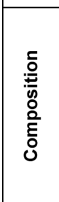 & 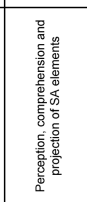 & 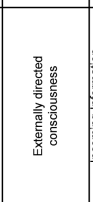 & 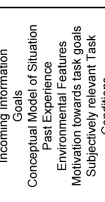 \\
\hline 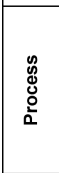 & 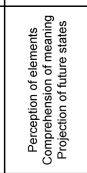 & 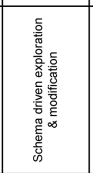 & 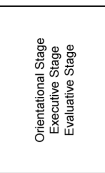 \\
\hline 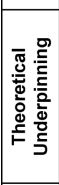 & 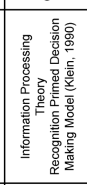 & 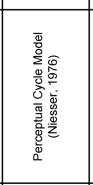 & 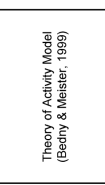 \\
\hline 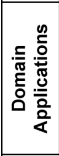 & 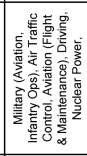 & 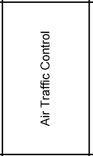 & $\frac{\mathscr{2}}{\frac{2}{2}}$ \\
\hline 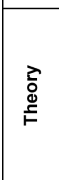 & 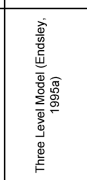 & 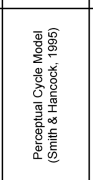 & 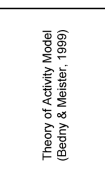 \\
\hline
\end{tabular}

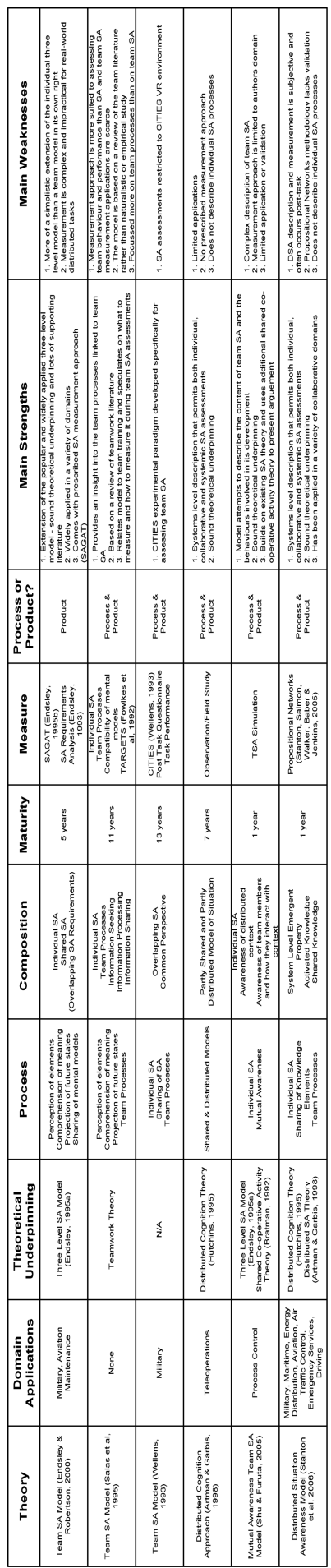




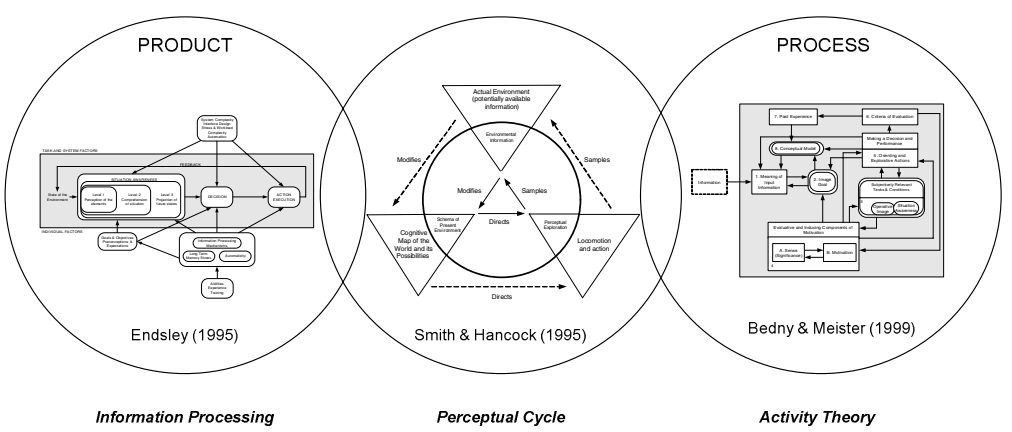

Fig. 1. Situation Awareness Models

Endsley has been criticized for using poorly understood approaches to underpin the model. Smith and Hancock (1995), for example, suggest that its reference to mental models, which themselves are ill-defined, is problematic. Similarly, Uhlarik \& Comerford (2002) criticise Endsley's theory for its use of an information-processing model that contains ill-defined psychological constructs that are subject to great debate themselves. The model has also been criticised for its inability to cope with the dynamic nature of SA (Stanton, Chambers \& Piggott, 2001; Uhlarik \& Comerford, 2002).

The perceptual cycle model (Smith and Hancock, 1995) has sound underpinning theory (Neisser, 1976) and is complete in that it refers to both the process (continuous sampling of the environment) and the product (continually updated schema) of SA. Unlike the three-level model, Smith and Hancock's model therefore grasps the dynamic nature of the construct. On the downside, the perceptual cycle model has not received anywhere near the attention that Endsley's model has, and its description of SA makes measuring the construct complex. Bedny \& Meister's description of the way in which SA dynamically modifies interaction with the world and then interaction with the world dynamically modifies SA is logical, and goes beyond the static perspective taken by Endsley's model. Despite this, there is a distinct lack of empirical evidence available to support their model, and consequently it has received less attention than Endsley's model.

In summary, each theory has its useful components. As Stanton et al (2001) point out, 'there appears to be an element of truth in all of them'. In terms of theoretical utility, Smith and Hancock's model is perhaps the most useful, since it caters for the dynamic aspects of SA (Stanton et al, 2001), whereas the theory of activity model is useful since it details an individuals internal activities involved when developing SA. Endsley's three level model, on the other hand, offers a very neat and intuitive description of SA which allows researchers to measure the construct simplistically and also to abstract SA requirements at each level. As a consequence of this, the three level model has been the most useful for researchers wishing to describe operator SA in complex systems and also for measuring the construct. Further, Endsley's work has proved to be the most useful for informing system design and evaluation (e.g. Endsley, Bolte \& Jones, 2003). One consistent theme that links these theories is that they describe SA acquisition and maintenance from the point of view of an individual operator and so fail to cater for team SA or SA during collaborative activity. 


\section{Situation Awareness in Collaborative Systems}

Salas, Prince, Baker and Shrestha (1995) point out that there is a lot more to team SA than merely combining individual team member SA. On a simple level we can say that team SA is multidimensional, comprising individual team member SA, shared SA between team members, and also the combined $S A$ of the whole team. Add to this the complexity of the systems in which team SA is critical (e.g. military command and control systems, emergency service domains etc), the various team processes involved in the development and maintenance of team SA (e.g. communication, co-ordination etc) and the various factors affecting team SA levels, and the complexity of the construct quickly becomes apparent. Based on a review of the literature Salas et al (1995) proposed a framework of team SA, suggesting that it comprises two critical, but poorly understood, processes: individual $S A$ and team processes. They subsequently defined team SA as "the shared understanding of a situation among team members at one point in time" (Salas et al, 1995). Other researchers have suggested that team SA refers to the level of overlap in SA between team members. Endsley (1989), for example, defines team SA as, "the degree to which every team member possesses the situation awareness required for his or her responsibilities" (Endsley, 1989) and shared SA as "the degree to which team members have the same SA on shared SA requirements" (Endsley \& Jones, 2001). Endsley (1989) suggests that, during team activities, SA can overlap between team members, in that individuals need to perceive, comprehend and project SA elements that are specifically related to their specific role in the team, but also elements that are required by themselves and by members of the team. Successful team performance therefore requires that individual team members have good SA on their specific elements and also the same SA for those elements that are shared (Endsley \& Robertson, 2000).

In conclusion to a review of team SA in aircraft maintenance teams, Endsley and Robertson (2000) suggested that good team SA is dependent on team members understanding the meaning of the information that is passed between one another. According to Endsley and Robertson (2000) this means that teams need to share pertinent data and the higher levels of SA, such as the significance of SA elements to the team's goals and also projected states. Endsley and Robertson (2000) go on to suggest that the primary factors linked to team performance are shared goals, the interdependence of team member actions, and the division of labour between team members. This means that some SA requirements are independent but also that team members possess shared goals and perform interdependent activities, which means that they also possess shared SA requirements (Endsley and Robertson (2000). Wellens (1993) suggests that the key to team SA lies in the arrangement of teams so that sufficient overlap between team member SA occurs to support co-ordination, but also so that sufficient separation between members allows individual SA acquisition. Wellens (1993) defines group or team SA as "the sharing of a common perspective between two or more individuals regarding current environmental events, their meaning and projected future".

In terms of how team SA is acquired and maintained, Bolstad \& Endsley (2000) suggested that the development of shared SA involves the following four factors: shared $S A$ requirements (e.g. the degree to which team members understand which 
information is needed by other team members), shared $S A$ devices (e.g. communications, shared displays and the shared environment), shared SA mechanisms (e.g. shared mental models), and shared SA processes (effective team processes for sharing relevant information). One of the key concepts thought to be critical to team SA is the notion of shared mental models. Fiore, Cuevas \& Salas (2003) suggest that a shared mental model is, "the activation in working memory of team and task-related knowledge while engaged in team interaction". It is thought that shared mental models facilitate communications between team members (Perla et al, 2000) and can allow team members to forecast the behaviour of other team members (Salas, Stout and Cannon Bowers, 1994). Endsley (1995a) even argues that team SA is more reliant on mental models than it is on verbal communication.

The role of team behaviours, such as team communication, co-ordination, adaptability, and co-operation and team attitudes, such as team trust, collective efficacy and orientation (Fiore et al, 2003) is often neglected when describing team SA. It seems logical to assume that an increased level of teamwork will lead to enhanced levels of team SA, however the specific relationship between team SA and team behaviours and attitudes remains largely unexplained. Most researchers have focused on communication as the key element in the acquisition of team SA. Nofi (2000) for example, singles out communication is the most critical element in the creation of team SA, whilst Entin and Entin (2000) suggest that communication is a prerequisite for high levels of team SA. Salas et al (1995) also highlighted the importance of communication in team SA acquisition.

The team SA theories identified in the literature are compared and contrasted in Table 2. The review leads us to conclude that there is a lack of a unified, universally accepted model of team SA. The approaches presented focus on either a shared awareness of the situation, sometimes called a 'common understanding' or on the overlap between team member SA requirements. It seems that there is a lack of a model that fully describes the processes involved, the content of team SA and also the factors impacting team SA. Based on a synthesis of the literature, it can be concluded that team SA comprises a team's compatible awareness of the situation. Team members must possess SA-related to their individual roles and goals within the team (some of which may be common or 'shared' with other team members), whilst also holding SA-related to other team members, including an awareness of other team members activities, roles, and responsibilities, and also to the team overall, including goals and performance. SA-related data and knowledge is distributed around the team through team processes such as communication, co-ordination and collaboration, and serves to inform and modify team member SA, which is also informed and modified by the overall teams SA. Thus a tripartite composition of team SA is apparent: individual team member $S A$ (some of which may be common or 'shared' with other team members), SA of other team members, and SA of the overall team. This simplistic view of team SA is presented in Figure 2.

The team or shared SA theory perspective is limited for a number of reasons (some of which are not alluded to here due to space limitations). Take, for example, military Networked Enabled Capability (NEC) scenarios. Such tasks involve numerous agents and artifacts working both collaboratively and in isolation from one another whilst being dispersed geographically, often over great distances. Viewing and assessing team SA in such environments is acutely complex. The dispersed, real world nature of 
tasks in these environments inhibits (or at least makes impractical) the use of objective probe techniques such as the Situation Awareness Global Assessment Technique (SAGAT; Endsley, 1995b). Also, the simplistic description of individual SA and shared SA can be viewed differently. For example, it may be that team members do not need to have SA of the overall team's situation, and that they only require SA for their specific task and role within the team, SA that is different but compatible with their counterparts. Therefore to suggest that all team members have their own SA and also shared SA with team members and of the overall team could be an oversimplification. Any cog in a machine does not need to 'know about' all of the other cogs, but it does need to be able to connect with adjacent cogs - thus we are suggesting that 'compatibility' is the key to team SA, rather than 'sharedness'. Any sharing of goals, intent, and understanding arises out of the need of the individual agents to perform their tasks and not for its own sake. We feel that the ideas of 'sharing' have mutated into a vague belief that sharing ensures a cohesive team, but we argue that 'compatibility' leads to cohesiveness.

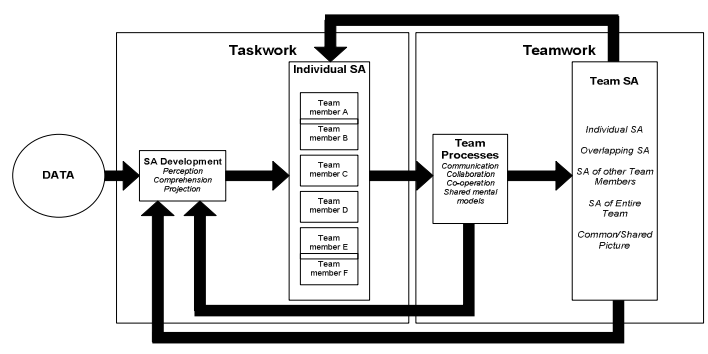

Fig. 2. Team SA

\section{Distributed Situation Awareness}

A more recent theme to emerge is the concept of distributed or systemic SA. Based upon distributed cognition theory (Hutchins, 1995), DSA approaches view SA as an emergent property (i.e. relationship between system elements) of collaborative systems. DSA is achieved through co-ordination between system units (Artman \& Garbis, 1998) and is viewed systemically rather than as an individual endeavor or shared understanding. Whilst recognising that individuals possess their own SA for a particular situation and that they share this understanding of the situation (Artman \& Garbis, 1998), DSA approaches assume that collaborative systems posses cognitive properties (such as SA) that are not part of individual cognition. No single member has the overall SA, rather it is distributed around the system. Artman and Garbis (1998) define team SA as,

"The active construction of a model of a situation partly shared and partly distributed between two or more agents, from which one can anticipate important future states in the near future" (Artman \& Garbis, 1998) 
Stanton, Stewart, Harris, Houghton, Baber, McMaster, Salmon, Hoyle, Walker, Young, Linsell, Dymott \& Green (2006) recently proposed a theory of DSA for complex systems. Stanton et al suggest that SA-related knowledge is distributed across the agents and artefacts comprising the system and that these knowledge 'themes' or 'topics', labelled knowledge elements, represent what agents 'need to know' in order to achieve success during task performance. In this case, the term knowledge represents the relationship between concepts (Shadbolt \& Burton, 1995) and refers to task-level knowledge, which relates to the goals and sub-goals of the task being performed. The ownership, usage and sharing of knowledge is dynamic and is dependent upon the task and its associated goals. Agents therefore have different SA for the same situation, but their SA can be overlapping, compatible and complementary, and deficiencies in one agents SA can be compensated by another agent. Stanton et al define DSA as 'activated knowledge for a specific task, at a specific time within a system'.

Stanton et al (2006) propose then, that a situation requires the use of appropriate knowledge (held by individuals, captured by devices etc.) that relates to the state of the environment and those changes as the situation develops. The 'ownership' of this knowledge is initially at the system, rather than individual level. This notion could be further extend to include 'meta-SA', where its knowledge of other agents' knowledge is contained in the system, such that each agent could potentially know where to go when they need to find something out. It is important to note that DSA approaches do not contend that individual SA perspectives are redundant; rather they provide an alternative, but complementary approach to viewing and describing SA in collaborative systems. For example, in extending the DSA to Endsley's three level model approach, we assume that within collaborative systems, some individuals are engaged in perception tasks, some are engaged in comprehension and in the projection tasks and others are engaged in response execution tasks.

\section{Discussion}

In this article we have attempted to present a synthesis of the literature on SA and team SA. The review indicated that the prominent SA models focus on SA acquisition and maintenance largely from the point of view of individual operators in complex systems. As a result of this, team SA as a theoretical construct remains a challenge to the human factors community. Team SA, at a very simplistic level, comprises individual team member SA, some of which may be common or shared, SA of other team members, and the holistic SA of the team as a whole. The development and maintenance of team SA is impacted by a myriad of factors, both at to the individual level (e.g. experience, stress, workload etc), team level (e.g. team behaviours such as communication and collaboration) and the systems level (e.g. system design, procedures, technology used etc). This multidimensional composition of team SA ensures that describing and assessing SA during real world collaborative tasks is complex. Despite the successful extension of Endsley's three level model to the team environment (Endsley and Robertson, 2000, Endsley and Jones, 2001), one still feels that, theoretically at least, team SA requires further investigation. Based on the review, it is concluded that recently emerged DSA approaches (e.g. Stanton et al, 
2006) are suited to describing and assessing SA in real world collaborative environments, or in environments where SA elements are not well defined a priori. Viewing SA in this way is fruitful for a number of reasons, including that it permits a systemic description of the knowledge comprising SA (which can be extrapolated to an individual SA level) and it allows judgements to be made on potential barriers to SA acquisition and maintenance. Further, considering SA in this way ensures that team SA is viewed in its entirety, rather than as its component parts (i.e. individual team member SA). In collaborative systems, tasks are rarely performed entirely independently of others, especially in complex situations and when critical decisionmaking is required (Artman \& Garbis, 1998) - these activities tend to require coordinated activity between several individuals (Cannon-Bowers and Salas, 1990; cited in Salas, Prince, Baker and Shrestha, 1995). It is important therefore that team SA assessments consider this co-ordination.

\section{References}

Artman, H., Garbis, C.: Situation awareness as distributed cognition. In: Proceedings of ECCE' 98, Limerick (1998)

Bedny, G., Meister, D.: Theory of activity and situation awareness. International Journal of Cognitive Ergonomics 3(1), 63-72 (1999)

Bolstad, C.A., Endsley, M.R.: The effect of task load and shared displays on team situation awareness. In: Proceedings of the 14th Triennial Congress of the International Ergonomics Association and the 44th Annual Meeting of the Human Factors and Ergonomics Society (2000)

Endsley, M.R.: Final report: Situation awareness in an advanced strategic mission (NOR DOC 89-32). Northrop Corporation, Hawthorne, CA (1989)

Endsley, M.R.: Towards a theory of Situation Awareness in Dynamic Systems. Human Factors 37, 32-64 (1995)

Endsley, M.R.: Measurement of Situation Awareness in Dynamic Systems. Human Factors 37, 65-84 (1995)

Endsley, M.R., Jones, W.M.: A model of inter- and intrateam situation awareness: Implications for design, training and measurement. In: McNeese, M., Salas, E., Endsley, M. (eds.) New trends in cooperative activities: Understanding system dynamics in complex environments, Human Factors and Ergonomics Society, Santa Monica, CA (2001)

Endsley, M.R., Robertson, M.M.: Situation awareness in aircraft maintenance teams. International Journal of Industrial Ergonomics 26, 301-325 (2000)

Endsley, M.R., Bolte, B., Jones, D.G.: Designing for situation awareness: An approach to human-centred design. Taylor and Francis, London (2003)

Entin, E., Entin, E.: Assessing Team Situation Awareness in Simulated Military Missions. Ergonomics for the New Millennium. In: Proceedings of the XIVth Triennial Congress of the International Ergonomics Association and 44th Annual Meeting of the Human Factors and Ergonomics Society, San Diego, California. vol. 1, pp. 73-76 (2000)

Fiore, S.M., Salas, E., Cuevas, H.M., Bowers, C.A.: Distributed coordination space: toward a theory of distributed team process and performance. Theoretical Issues in Ergonomics Science 4, 340-364 (2003)

Fracker, M.: Measures of Situation Awareness: Review and Future Directions (Rep. No.ALTR-1991-0128). Wright Patterson Air Force Base, Ohio: Armstrong Laboratories, Crew Systems Directorate. (1991) 
Hutchins, E.: Cognition in the wild. MIT Press, Cambridge Massachusetts (1995)

Niesser, U.: Cognition and reality: Principles and implications of cognitive psychology. Freeman, San Francisco (1976)

Nofi, A.: Defining and Measuring Shared Situational Awareness, DARPA (2000), http://www.thoughtlink.com/publications/DefiningSSA00Abstract.htm.

Perla, P., Markowitz, M., Nofi, A., Weuve, C., Loughran, J., Stahl, M.: Gaming and Shared Situation Awareness. DARPA GamingSSA00Abstract.htm (2000), http://www.thoughtlink.com/publications/

Salas, E., Stout, R.J., Cannon-Bowers, J.A.: The role of shared mental models in developing shared situational awareness. In: Gilson, D., Garland, D.J., Koonce, J.M. (eds.) Situational Awareness in Complex Systems: proceedings of a CAHFA conference, Embry-Riddle Aeronautical University Press, Daytona Beach, FL (1994)

Salas, E., Prince, C., Baker, P.D., Shrestha, L.: Situation awareness in team performance. Human Factors 37(1), 123-126 (1995)

Shu, Y., Furuta, K.: An inference method of team situation awareness based on mutual awareness. Cognition Technology \& Work. 7, 272-287 (2005)

Shadbolt, N.R., Burton, M.: Knowledge elicitation: A systemic approach. In: Wilson, J.R., Corlett, E.N. (eds.) Evaluation of Human Work: A Practical Ergonomics Methodology, pp. 406-440 (1995)

Smith, K., Hancock, P.A.: Situation awareness is adaptive, externally directed consciousness. Human Factors 37(1), 137-148 (1995)

Stanton, N.A., Chambers, P.R.G., Piggott, J.: Situational Awareness and safety. Safety Science 39, 189-204 (2001)

Stanton, N.A., Stewart, R., Harris, D., Houghton, R.J., Baber, C., McMaster, R., Salmon, P., Hoyle, G., Walker, G.H., Young, M.S., Linsell, M., Dymott, R. Green, D.A.: Distributed situation awareness in dynamic systems: theoretical development and application of an ergonomics methodology. Ergonomics.49, 12-13, 1288-1311 (2006)

Uhlarik, J., Comerford, D.A.: A review of situation awareness literature relevant to pilot surveillance functions (DOT/FAA/AM-02/3). Department of Transportation. Federal Aviation Administration, Washington, DC, U.S (2002)

Wellens, A.R.: Group situation awareness and distributed decision making: From military to civilian applications. In: Castellan, N.J. (ed.) Individual and Group Decision Making: Current Issues, pp. 267-287. Erlbaum Associates, Mahwah (1993) 